REVIEW

\title{
Who should have a sleep study for sleep related breathing disorders?
}

\author{
L Whiteford, P Fleming, A J Henderson
}

Arch Dis Child 2004;89:851-855. doi: 10.1136/adc.2003.036178

By the time children are 5 years old more of their life will have been spent asleep than awake. Sleep medicine is therefore especially relevant in paediatrics. However, one recent survey showed that paediatricians' knowledge of sleep medicine is poor and that few paediatricians enquire about sleep as part of their general consultation.'

$\mathrm{V}$ entilatory function is physiologically reduced during sleep in adults and children. Therefore, respiratory disorders are exacerbated and may only be clinically apparent during sleep. Assessing respiratory status only when a child is awake will underestimate the severity of sleep related breathing disorders or miss them altogether. Such failure to recognise sleep related breathing disorders can have significant adverse consequences on children's physical health and on their behaviour and learning.

\section{HOW ARE SLEEP RELATED PROBLEMS IDENTIFIED?}

The first step towards identifying a sleep related breathing disorder is to take a careful history. Symptoms during sleep and wakefulness should be enquired about as part of the general paediatric consultation (table 1).

Symptoms of sleep disturbance are commonly reported by parents and include behavioural problems, parasomnias, and insomnia. ${ }^{2}$ The commonest symptom associated with possible sleep disordered breathing is snoring, which may occur occasionally, only in association with upper respiratory infection or seasonal rhinitis, or on the majority of nights, termed habitual snoring. A history of snoring may be accompanied by reports of apnoea or dyspnoea associated with cessation of the respiratory noise. Such events are frightening to parents who may then maintain increased vigilance over their child during the night. Restless sleep with frequent changes of body position may indicate subcortical arousals. ${ }^{3}$ Children may sometimes adopt unusual sleeping postures, such as hyperextension of the neck to increase airway patency. Autonomic disturbances, including heart rate variability, ${ }^{4}$ profuse sweating, and nocturnal enuresis, ${ }^{5}$ have been reported in children with sleep disordered breathing.

Children with breathing disturbances during sleep may be difficult to rouse or irritable on waking. Hypoventilation during sleep can be associated with the presence of morning head- aches, vomiting, and lack of appetite for breakfast. Daytime symptoms include mouth breathing in children with adenoidal hypertrophy. Excessive daytime sleepiness is less common in children than in adults with sleep disordered breathing. ${ }^{6}$ However, behavioural disturbances such as hyperactivity or aggression may occur, ${ }^{7}$ and chronic sleep disturbance is associated with learning difficulties, ${ }^{9}$ developmental delay, failure to thrive, and cardiovascular dysfunction. Populations of children at increased risk of sleep related breathing disorders include those with adenotonsillar hypertrophy, craniofacial abnormalities, obesity, chronic respiratory or chest wall disease, and neuromuscular disorders affecting upper airway control and respiratory muscle function.

Physical examination should include assessment of factors predisposing to sleep related breathing disorders and of potential consequences of sleep disordered breathing. The former will include careful examination of the upper airway, looking particularly for signs of adenotonsillar hypertrophy, the commonest cause of increased upper airway resistance during sleep in children. Nasal patency should be assessed and the presence of craniofacial and palatal abnormalities noted. These include syndromes that affect the development of the mid-face and mandible, such as Pierre Robin, Apert, Treacher Collins, CHARGE, Goldenhar, and Pfeiffer, ${ }^{10}$ and conditions that may be associated with multifactorial sleep related breathing disturbances, for example achondroplasia, ${ }^{11}$ in which central and obstructive apnoeas and chest wall restriction can all contribute to sleep related breathing disturbances. Children who have had palatoplasty for cleft palate may also have narrow posterior airways and be at risk of obstructive sleep apnoea. ${ }^{12}$ Respiratory examination, including measurement of respiratory function in appropriately aged children with suspected pulmonary or chest wall disease should be performed. The contribution of neuromuscular diseases to sleep disordered breathing should be considered in children with disorders affecting muscle strength and tone, including cerebral palsy, congenital and acquired myopathies and muscular dystrophies, particularly in association with kyphoscoliosis, and disorders affecting control of upper airway tone and reflexes, such as bulbar or pseudobulbar palsy.

Abbreviations: $\mathrm{CCHS}$, congenital central hypoventilation syndrome; OSAS, obstructive sleep apnoea syndrome; PSG, polysomnography; REM, rapid eye movement; UARS, upper airway resistance syndrome 
Table 1 Reported features that may suggest a sleep related breathing disorder

\begin{tabular}{ll}
\hline Features during sleep & Snoring \\
& Witnessed apnoeas \\
& Cyanosis \\
& Parental concern about their child's breathing \\
& Restless sleep \\
& Unusual sleeping posture \\
Symptoms on waking & Difficult to rouse in the morning \\
& Irritability/bursts of anger on waking \\
& Morning headaches or vomiting \\
& Lack of appetite for breakfast \\
Daytime features & Mouth breathing \\
& Excessive daytime sleepiness \\
& Hyperactivity/behavioural disturbance \\
& Learning/memory difficulties \\
& Developmental delay \\
& Poor growth \\
\hline
\end{tabular}

Potential complications of sleep disordered breathing include failure of expected growth. ${ }^{13}$ Cardiac examination is usually normal, although signs of pulmonary hypertension may be present. Systemic hypertension is probably less common in children than in adults with obstructive sleep apnoea but has been described. ${ }^{14}{ }^{15}$ Cor pulmonale was described in earlier studies but is uncommonly seen, possibly due to earlier recognition and intervention of severe obstructive sleep apnoea.

Although clearly important, clinical history and examination alone are unable to differentiate between children with and without significant sleep related breathing disorders. Symptoms are common; the prevalence of habitual snoring in a general population of children is $10 \%{ }^{16}$ However, most habitual snorers will have primary snoring, characterised by the absence of associated hypoxaemia, hypercapnia, sleep disruption, or daytime symptoms. This is a benign condition that does not require treatment. Only a small proportion of children who habitually snore have obstructive sleep apnoea requiring treatment. Therefore, attempts have been made to devise screening methods to identify children at risk of significant sleep related breathing disorders.

Brouillette and others ${ }^{17}$ designed a clinical scoring system based on three questions about breathing during sleep. When applied to a general paediatric population, this was able to discriminate between healthy children and those with severe obstructive sleep apnoea syndrome (OSAS) but did not identify those with mild to moderate OSAS. Carroll and coworkers ${ }^{18}$ extended this scoring system and applied it to a population of children referred to a sleep clinic for assessment of snoring or difficulty breathing during sleep. In this setting it was not possible to reliably distinguish primary snoring from OSAS using the clinical history alone. Preutthipan and others ${ }^{19}$ studied parents' ability to predict the severity of childhood OSAS based on observation of their child's sleep. They showed that parents of children with severe OSAS were more likely to report cyanosis, obstructive apnoeas, and extremely loud snoring. They felt the need to shake or watch their child more frequently during sleep and were afraid of their child's breathing at night. However, no single parental observation or combination of these could be used to confidently predict severe OSAS. Other investigators have also shown that clinical evaluation alone is not sufficient to identify children with significant sleep related breathing difficulties. ${ }^{20} 21$ A group at high risk for severe obstructive sleep apnoea can be identified but milder degrees may be missed. Therefore, some form of sleep study is advisable for further evaluation of symptomatic cases.

\section{WHAT INVESTIGATIONS ARE AVAILABLE?}

Overnight polysomnography (PSG) in a sleep laboratory is considered to be the gold standard investigation for suspected abnormal breathing during sleep in children. A dedicated paediatric sleep laboratory, which can accommodate the unique needs of children, is the ideal setting for such investigations. Studies in adult laboratories may be suboptimal and will be misinterpreted if adult diagnostic standards are applied. Despite the fact that young children take frequent naps, PSG is best performed at night during natural sleep. Marcus and others ${ }^{22}$ reported that nap studies had a positive predictive value of $100 \%$, but a negative predictive value of only $20 \%$ for sleep disordered breathing. Nap studies can be useful for screening but are of limited diagnostic value, as they may not include episodes of rapid eye movement (REM) sleep; the behavioural state during which abnormalities are most likely to be seen. There is also some evidence that a sleep study is more likely to be abnormal in the later part of the night, which would be missed by a nap study. Sedatives should not be used to induce sleep for PSG as they depress upper airway dilator muscle function and may induce worse apnoea in children predisposed to OSAS. ${ }^{23}$

PSG simultaneously records a number of physiological variables, which can be selected depending on the clinical context in which the study is being carried out. They typically include respiratory movements, gas exchange, cardiac rate and rhythm, sleep state, and muscle activity. Respiratory effort can be assessed by simultaneously recording chest and abdominal wall movement using inductance plethysmography. Paradoxical inward rib cage movement (PIRCM) during inspiration is associated with upper airway obstruction during sleep. ${ }^{24}$ However, the usefulness of this measurement is limited in infants and young children, in whom paradoxical rib cage movements occur physiologically during REM sleep. A more accurate way of measuring respiratory effort utilises oesophageal pressure monitoring, but this is more invasive and the presence of an oesophageal manometer can interfere with sleep, making it unacceptable to many children. Airflow at the nose and mouth are seldom measured directly but proxy measures indicating the presence of gas flow, such as temperature change measured with a thermistor, can detect apnoeas. Oxygenation is measured by pulse oximetry, and carbon dioxide tensions can be measured non-invasively using an end-tidal $\mathrm{CO}_{2}$ catheter or transcutaneous $\mathrm{CO}_{2}$ electrode.

Sleep staging ensures that the study includes periods of REM sleep. It also allows arousals from sleep, including subcortical arousals, to be identified, and hence provides information on sleep quality. Staging sleep involves the combined measurement of the electroencephalogram (EEG), electro-oculogram (EOG) to record rapid eye movements, and the electromyogram (EMG) to record submental and tibial muscle activity, thus allowing movement arousals to be detected. There is some debate about the value of precise sleep staging in the diagnosis of straightforward conditions such as obstructive sleep apnoea in an otherwise healthy child with adenoidal hypertrophy, in which case quiet sleep can be differentiated from active sleep using cardiorespiratory and behavioural variables. Additionally, advances in technology, such as neural network analysis, ${ }^{25}$ have the potential to simplify this process.

An audio video recording during sleep is usually performed as part of the PSG as it is helpful in correlating physiological disturbances with clinical or behavioural findings. A trained technician is required to supervise the study to assure quality. Therefore, PSG is expensive and time consuming to perform. Additionally, there are limited resources for full polysomnography in children in the United Kingdom and other 
countries. Therefore, researchers have been prompted to evaluate other options for the assessment of sleep disordered breathing either as diagnostic or screening investigations.

Pulse oximetry is the most widely used of the available screening methods. In some circumstances it can be useful, but it does have significant limitations. The finding of intermittent episodes of hypoxaemia in a snoring child is highly suggestive of OSAS. Stradling and others showed that pulse oximetry had $93 \%$ sensitivity and $86 \%$ specificity for detecting OSAS. ${ }^{26}$ Brouillette and others ${ }^{27}$ showed a high positive predictive value for pulse oximetry but also a high false negative rate. Sleep related breathing problems are more likely to occur during rapid eye movement (REM) than during non-REM sleep. It is not possible to determine if adequate periods of REM sleep have been included in a sleep study using pulse oximetry alone. Some children who snore have significant disturbance in their breathing and sleep without recurrent episodes of hypoxaemia. There is evidence that continuous partial airway obstruction may be the most common presentation of disordered breathing during sleep in children of all ages, ${ }^{28}{ }^{29}$ and this will not be detected by pulse oximetry. Additionally, pulse oximetry is not a reliable indicator of severity of OSAS. ${ }^{30}$ This is potentially important in preoperative assessment, particularly of high risk children with adenotonsillar hypertrophy who have an increased risk of perioperative complications with severe disease. Pulse oximetry does however have an important role in assessing oxygenation during sleep in children with chronic lung disease and neuromuscular disorders.

Audio and video taping have also been used to screen for sleep disordered breathing. Recording the snoring sound can be used to detect apnoeas, but it cannot differentiate between central and obstructive apnoea. ${ }^{31}$ Goldstein and colleagues ${ }^{32}$ found that a sound recording was positive when compared to PSG only $50 \%$ of the time. Lamm and others ${ }^{33}$ found that home audiotapes were not sufficiently specific to reliably distinguish primary snoring from OSAS. A videotape of a child during sleep can provide useful information. It allows the physician an opportunity to observe disturbed sleep. However it is not practical in a clinical context to record and view more than a limited portion of the sleep period. The severity of sleep disordered breathing may vary throughout the night. The design of sophisticated computer systems for analysing video material may circumvent this problem. Combining audiotaping with pulse oximetry adds to the diagnostic value; ${ }^{31}$ however, lack of arousal data and information to stage sleep remains a problem.

Due to the limitations of single channel recording systems, attempts have been made to develop simple multichannel devices that are suitable for unattended home use or with minimal supervision. There is limited evidence of the performance of these devices in children to date, but recent studies of such monitors compared in both laboratory and home conditions with PSG, have shown that obstructive apnoea/hypopnoea syndrome in adults can be diagnosed satisfactorily in a substantial proportion of cases. ${ }^{34}$ Although such comparisons do not exist for children, unattended home PSG has been evaluated in a research context and found to provide acceptably high quality data. ${ }^{35}$ Therefore, this represents a potentially useful and cost effective approach to the diagnosis of obstructive apnoea in children that deserves further evaluation.

\section{WHAT ABNORMALITIES CAN BE DETECTED? Obstructive sleep apnoea and upper airway resistance syndromes}

The most common indication for performing sleep studies in children is for the detection of obstructive sleep apnoea syndrome (OSAS). OSAS in children is characterised by recurrent events of partial or complete airway obstruction during sleep, resulting in disruption in normal ventilation and sleep patterns, ${ }^{36}$ but rarely with prolonged apnoea. Episodes of obstruction occur primarily during REM sleep, in contrast with obstructive apnoeas in adults, which are mostly non-REM phenomena. The prevalence of OSAS in preschool children is estimated to be $1-3 \% .^{37} 38$

The majority of cases of OSAS in children are associated with adentonsillar hypertrophy. It is most commonly diagnosed between the ages of 2 and 8 years when the tonsils and adenoids are largest in relation to the upper airway size. However, the symptoms of OSAS are not simply related to structural narrowing of the airway by physical obstruction. This is supported by studies which have shown no correlation between upper airway or adenotonsillar size and OSAS, ${ }^{39} 40$ and by studies in which children have failed to show improvement after adenotonsillectomy despite the lack of other risk factors for OSAS. ${ }^{21}$ A combination of structural and neuromotor abnormalities is likely to be important for OSAS to develop. The role that these two factors have will vary in different individuals. In children with adenotonsillar hypertrophy, structural factors will tend to predominate, although subtle neuromotor abnormalities are likely also to be present. ${ }^{41}$ In children with cerebral palsy and neurodisability, it is more likely that neuromotor factors will be to the fore.

The classical picture of OSAS in which snoring is associated with obstructive apnoea and hypoxaemia represents only a small proportion of children with sleep disordered breathing. It is now widely recognised that a spectrum of abnormalities exists $^{42}$ between primary snoring and classical OSAS, with two further conditions, upper airway resistance syndrome and obstructive hypoventilation being intermediate between the extremes. It is also recognised that children may move up or down this spectrum in response to seasonal variations and with age or growth. Therefore, these should not be regarded as static conditions. Upper airway resistance syndrome (UARS) is characterised by increased upper airway resistance and increased work of breathing during sleep sufficient to cause frequent microarousals (brief arousals $<15$ seconds) from sleep, which lead to excessive daytime sleepiness and diminished neurocognitive function. In obstructive hypoventilation, increased airway resistance sufficient to cause partial airways obstruction and hypoventilation occurs, leading to peak end tidal $\mathrm{CO}_{2}>55 \mathrm{~mm} \mathrm{Hg}$ or end tidal $\mathrm{CO}_{2}>45$ mm HG for more than $60 \%$ of the total sleep time. Snoring is common to all of these conditions. UARS and obstructive hypoventilation are not detectable using single channel screening, such as oximetry, but multichannel systems have the potential to identify obstructive hypoventilation and may also be useful in screening of UARS, the clinical significance of which has yet to be fully determined in children.

\section{Other conditions}

In children with chronic lung disease and limited pulmonary reserve, such as infants with chronic lung disease of prematurity and children with cystic fibrosis, the normal effects of sleep on respiratory function can result in significant ventilatory and gas exchange abnormalities. ${ }^{43}$ Children may require higher inspired oxygen concentrations during sleep, whether or not supplemental oxygen is needed during wakefulness. These children can be identified by continuous assessment of oxygen saturation during sleep.

Children with neuromuscular disease, especially those with progressive conditions such as Duchenne muscular dystrophy, will eventually develop respiratory disturbances during sleep. ${ }^{44}$ These are most pronounced during REM sleep due to diminished respiratory drive, atonia of the upper airway and intercostal muscles, and dependence of respiration on diaphragmatic function. Both obstructive apnoea and 
alveolar hypoventilation can occur. Progressive scoliosis is often a contributing factor in these children. Mild hypercapnia and REM sleep desaturation are the first abnormalities to be observed and can be detected by combined recordings of gas exchange using oximetry and end tidal $\mathrm{CO}_{2}$. Treatment is limited to supportive measures but these can prolong the duration and, most importantly, improve the quality of life for these patients.

Sleep studies will also identify conditions in which there is an underlying abnormality in the central control of respiration. These conditions may be primary, such as congenital central hypoventilation syndrome (CCHS), or secondary to diseases of the spinal cord or brain stem. CCHS may range in severity from mild alveolar hypoventilation during sleep with adequate ventilation during wakefulness to complete apnoea during sleep and severe hypoventilation even when awake. Infants usually present with cyanosis and respiratory failure, or occasionally apnoea at birth. Rarely, infants present later with apparent life threatening events or cor pulmonale. Definitive diagnosis requires careful evaluation using PSG, including measurement of ventilation during wakefulness, REM, and non-REM sleep states. Treatment of CCHS is with long term support of respiration. As ventilation requirements will change over time in such patients it is necessary that PSG should be repeated at regular intervals. It is our practice to do so on a six monthly basis.

\section{CONSEQUENCES OF SLEEP DISORDERED BREATHING IN CHILDREN}

Failure to recognise sleep disordered breathing in children can have significant consequences on growth, cardiac, and neurological function. Early surveys of children with severe OSAS reported failure to thrive in $27-56 \%$ of cases. ${ }^{13} 45$ Increased recognition of OSAS and earlier intervention has made failure to thrive the exception in recent times. However, children with OSAS still tend to have a growth spurt following adenotonsillectomy. ${ }^{46}$ This would appear to be due to decreased work of breathing postoperatively rather than to increased caloric intake. ${ }^{47}$ Two studies have suggested that endocrine factors may also have a role. Increases in insulin-like growth factor (IGF-1) and its binding protein have been shown after adenotonsillectomy in children with OSAS. $^{48} 49$

Recurrent nocturnal hypoxemia, hypercapnia, and respiratory acidosis can lead to pulmonary hypertension and on to cor pulmonale and congestive heart failure. This used to be a common presentation of OSAS in childhood but is now rarely reported. However, asymptomatic degrees of pulmonary hypertension may be more common than previously appreciated. Tal and others have shown reduced right ventricular ejection fractions in $37 \%$ of children with OSAS, although only $7 \%$ had clinical evidence of pulmonary hypertension. ${ }^{50}$ All these children showed normalisation of heart function after surgery. Routine screening of children with suspected OSAS with ECG or echocardiogram is unlikely to be worthwhile.

Systemic hypertension is a common complication of OSAS in adults but less frequently reported in children. Marcus and colleagues found that children with OSAS had a significantly higher systemic diastolic blood pressure (but no difference in systolic pressure) than children with primary snoring. ${ }^{14}$ This observation raised concerns about the long term consequences to adult health of albeit mild increase of blood pressure during childhood associated with OSAS.

Children with sleep disordered breathing have been shown to have a high incidence of neurocognitive and behavioural disturbances ${ }^{51-54}$ These include attention disorders, memory and learning disabilities, school failure, developmental delay, hyperactivity, aggressiveness, and withdrawn behaviour. ${ }^{8}$
Behavioural symptoms may be the primary clinical manifestation of sleep disordered breathing. Guilleminault and others showed that children aged 6 years and over who were referred to a sleep disorders clinic were frequently seen by the school counsellor before coming to the clinic, and concerns about attention deficit disorder and special educational needs had been raised. ${ }^{52}$ Rebellious behaviour was encountered more often than frank sleepiness. O'Brien and colleagues identified a high prevalence of sleep disordered breathing in children aged 5-7 years with mild attention deficit hyperactivity disorders (ADHD) compared with children with severe ADHD or none. ${ }^{53}$

Further evidence for a link between behaviour and neurological symptoms and sleep disordered breathing comes from studies that have shown improvement in these symptoms after treatment with adenotonsillectomy. ${ }^{84}$ Gozal performed screening for sleep disordered breathing in first grade students who were performing in the lowest (10th) centile of their class. He found that a surprisingly high proportion (18\%) were snorers with associated nocturnal gas exchange abnormalities. Children treated with adenotonsillectomy showed improvement in their grades the following year, whereas those left untreated did not.

\section{GUIDELINES FOR REFERRAL AND INVESTIGATION OF SUSPECTED SLEEP DISORDERED BREATHING IN CHILDREN}

The American Academy of Pediatrics has recently published clinical practice guidelines on diagnosis and management of OSAS in children. ${ }^{55}$ Although there are currently gaps in the evidence, the following is suggested as an approach to the recognition of OSAS in children:

(1) The possibility of obstructive sleep apnoea should be considered in children with habitual snoring, although the majority of these children will have primary snoring. Although clinical assessment is poor at discriminating primary snoring from OSAS, symptoms or signs suggestive of the latter should prompt further investigation. In the presence of significant complications, such as cardiorespiratory failure, investigation should be urgent in a specialist centre.

(2) Single channel systems, such as overnight oximetry, may be helpful if they show positive results in the context of a suggestive history of uncomplicated OSAS. However, a normal study does not exclude OSAS and polysomnography remains the gold standard investigation. Unattended home studies using multichannel systems appear promising and deserve further evaluation in this context.

(3) Complex and high risk patients, such as those with craniofacial abnormalities, neuromuscular disorders, and suspected central hypoventilation, should be referred to a specialist centre for further investigation.

\section{Authors' affiliations}

L Whiteford, P Fleming, A J Henderson, Bristol Royal Hospital for Children, Department of Paediatric Respiratory Medicine \& Sebastian Diamond Mother \& Baby Sleep Laboratory, Bristol, UK

\section{REFERENCES}

1 Owens JA. The practice of paediatric sleep medicine: results of a community survey. Pediatrics 2001;108:e51.

2 Archbold KH, Pituch KJ, Panahi P, et al. Symptoms of sleep disturbances among children at two general pediatric clinics. J Pediatr 2002;140:97-102.

3 Mograss MA, Ducharme FM, Brouillette RT. Movement/arousals. Description, classification and relationship to sleep apnea in children. Am J Respir Crit Care Med 1994;150:1690-6. 
4 Baharav A, Kotagal S, Rubin BK, et al. Autonomic cardiovascular control in children with obstructive sleep apnea. Clin Auton Res 1999;9:345-51.

5 Brooks LJ, Topol HI. Enuresis in children with sleep apnea. J Pediatr 2003:142:515-18.

6 Carroll JL, Loughlin GM. Diagnostic criteria for obstructive sleep apnea syndrome in children. Pediatr Pulmonol 1992;14:71-4.

7 Dahl RE. The impact of inadequate sleep on children's daytime cognitive function. Semin Pediatr Pulmonol 1996;3:44-55.

8 Gozal D. Sleep disordered breathing and school performance in children. Pediatrics 1998;102:616-20.

9 Urschitz MS, Guenter A, Eggebrecht E, et al. Snoring, intermittent hypoxia and academic performance in primary schoolchildren. Am J Respir Crit Care Med 2003;168:464-8

10 Perkins JA, Sie KCY, Milczuk H, et al. Airway management in children with craniofacial abnormalities. Cleft Palate Craniofac J 1997;34:135-40.

11 Mogayzel PJ, Carroll JL, Loughlin GM, et al. Sleep disordered breathing in children with achondroplasia. J Pediatr 1998;132:667-71.

12 Rose E, Thissen U, Otten JE, et al. Cephalometric assessment of the posterior airway space in patients with cleft palate after palatoplasty. Cleft Palate Craniofac J 2003;40:498-503.

13 Brouillette RT, Fernbach SK, Hunt CE. Obstructive sleep apnea in infants and children. J Pediatr 1982;100:31-40.

14 Marcus CL, Greene MG, Carroll JL. Blood pressure in children with obstructive sleep apnea. Am J Respir Crit Care Med 1998;157:1098-103.

15 Enright PL, Godwin JL, Sherril DL, et al. Blood pressure elevation associated with sleep related breathing disorder in a community sample of white and Hispanic children: the Tucson Children's Assessment of Sleep Apnea Study. Arch Pediatr Adolesc Med 2003;157:901-4.

16 Ali NJ, Stradling JR. Epidemiology and natural history of snoring and sleepdisordered breathing in children. In: Loughlin GM, Carroll JL, Marcus CL, eds. Sleep and breathing in children. A developmental approach. New York: Marcel Dekker, 2000.

17 Brouillette R, Hanson D, David R, et al. A diagnostic approach to suspected obstructive sleep apnea in children. J Pediatr 1984;105:10-14.

18 Carroll JL, McColley SA, Marcus CL, et al. Inability of clinical history to distinguish primary snoring from obstructive sleep apnea syndrome in children. Chest 1995;108:610-18.

19 Preutthipan A, Chantarojanasiri T, Suwanjutha S, et al. Can parents predict the severity of childhood obstructive sleep apnoea? Acta Paediatr 2000;89:708-12.

20 Wang RC, Elkins TP, Keech D, et al. Accuracy of clinical evaluation in pediatric obstructive sleep apnea. Otolaryngol Head Neck Surg 1998; 1 18:69-73.

21 Suen JS, Arnold JE, Brooks JL. Adenotonsillectomy for treatment of obstructive sleep apnea in children. Arch Otolarngol Head Neck Surg 1995; 121:525-30.

22 Marcus CL, Keens TG, Ward SL. Comparison of nap and overnight polysomnography in children. Pediatr Pulmonol 1992;13:16-21.

23 Biban P, Baraldi E, Pettennazzo A, et al. Adverse effects of chloral hydrate in two young children with obstructive sleep apnea. Pediatrics 1993;92:461-3.

24 Kohyama J, Shiiki T, Shimohira M, et al. Asynchronous breathing during sleep. Arch Dis Child 2001;84:174-7.

25 Shimada T, Shiina T, Saito Y. Detection of characteristic waves of sleep EEG by neural network analysis. IEEE Trans Biomed Eng 2000;47:369-79.

26 Stradling JR, Thomas G, Warley AR, et al. Effect of adenotonsillectomy on hypoxaemia, sleep disturbance and symptoms in snoring children. Lancet 1990;335:249-53.

27 Brouillette RT, Morielli A, Leimanis A, et al. Nocturnal pulse oximetry as an abbreviated testing modality for paediatric obstructive sleep apnea. Pediatrics 2000; 105:405-12

28 Rosen CL, D'Andrea L, Haddad GG. Adult criteria for obstructive sleep apnea do not identify children with serious obstruction. Am Rev Respir Dis 1992; 146:1231-4.

29 Guilleminault C, Pelayo R, Leger D, et al. Recognition of sleep disordered breathing in children. Pediatrics 1996;98:871-82.

30 Rosen GM, Muckle RP, Mahowald MW, et al. Postoperative respiratory compromise in children with obstructive sleep apnea: can it be anticipated? Pediatrics 1994;93:784-8.

31 White JE, Smithson AJ, Close PR, et al. The use of sound recording and oxygen saturation in screening snorers for obstructive sleep apnea. Clin Otolarngol 1994; 19:218-21.
32 Goldstein NA, Sculerati N, Walsleben JA, et al. Clinical diagnosis of pediatric obstructive sleep apnea validated by polysomnography. Otolaryngol Head Neck Surg 1994;111:611-17.

33 Lamm C, Mandeli J, Kattan M. Evaluation of home audiotapes as an abbreviated test for obstructive sleep apnea syndrome (OSAS) in children. Pediatr Pulmonol 1999;27:267-72.

34 Dingli K, Coleman EL, Vennelle M, et al. Evaluation of a portable device for diagnosing the sleep apnoea/hypopnoea syndrome. Eur Respir J 2003;21:253-9.

35 Goodwin JL, Enright PL, Kaemingk KL, et al. Feasibility of using unattended polysomnography in children for research: report of the Tucson Children's Assessment of Sleep Apnea study (TuCASA). Sleep 2001;24:937-44.

36 American Thoracic Society. Cardiorespiratory sleep studies in children. Establishment of normative data and polysomnographic predictors of morbidity. Am J Respir Crit Care Med 1996;160:1381-7.

37 Ali NJ, Pitson DJ, Stradling JR. Snoring, sleep disturbance and behaviour in 4-5 year old children. Arch Dis Child 1993;68:360-6.

38 Gislason T, Benediktsdottir B. Snoring, apneic episodes and nocturnal hypoxemia among children 6 months to 6 years old. An epidemiologic study of lower limit of prevalence. Chest 1995; 107:963-6.

39 Fernbach SK, Brouillette RT, Riggs TW, et al. Radiologic evaluation of adenoids and tonsils in children with obstructive apnea: plain films and fluoroscopy. Pediatr Radiol 1983;13:258-65.

40 Mahboubi S, Marsh RR, Potsic WP, et al. The lateral neck radiograph in adenotonsillar hyperplasia. Int J Pediatr Otorhinolaryngol 1985;10:67-73.

41 Marcus CL, McColley SA, Carroll JL, et al. Upper airway collapsibility in children with obstructive sleep apnea syndrome. J Appl Physiol 1994:77:918-24.

42 Loughlin GM. Obstructive sleep apnea syndrome in children. Diagnosis and management. In: Loughlin GM, Carroll JL, Marcus CL, eds. Sleep and breathing in children. A developmental approach. New York: Marcel Dekker, 2000.

43 Gaultier C. Effects of breathing during sleep in children with chronic lung disease. In: Loughlin GM, Carroll JL, Marcus CL, eds. Sleep and breathing in children. A developmental approach. New York: Marcel Dekker, 2000.

44 Givan DC. Sleep and breathing in children with neuromuscular disease. In: Loughlin GM, Carroll JL, Marcus CL, eds. Sleep and breathing in children. A developmental approach. New York: Marcel Dekker, 2000.

45 Guilleminault C, Korobkin R, Winkle R. A review of 50 children with obstructive sleep apnea syndrome. Lung 1981;159:275-87.

46 Williams EF, Woo P, Miller R, et al. The effects of adenotonsillectomy on growth in young children. Otolaryngol Head Neck Surg 1991;104:509-16.

47 Marcus CL, Carroll JL, Koerner CB, et al. Determinants of growth in children with obstructive sleep apnea syndrome. J Pediatr 1994;125:556-62.

48 Bar A, Tarasiuk A, Segev Y, et al. The effect of adenotonsillectomy on serum insulin-like growth factor-I and growth in children with obstructive sleep apnea syndrome. J Pediatr 1999;135:76-80.

49 Nieminen $\mathbf{P}$, Lopponen T, Tolonen U, et al. Growth and biochemical markers of growth in children with snoring and obstructive sleep apnea. Pediatrics 2002; 109:e55.

50 Tal A, Leiberman A, Margulis, et al. Ventricular dysfunction in children with obstructive sleep apnea syndrome: radionuclide assessment. Pediatr Pulmonol 1988:4:139-43.

51 Ali NJ, Pitson D, Stradling JR. Natural history of snoring and related breathing behaviour problems between the ages of 4 and 7 years. Arch Dis Child 1994;71:74-6.

52 Guilleminault C, Pelayo R. Neuropyschological consequences of disordered breathing during sleep. In: Loughlin GM, Carroll JL, Marcus CL, eds. Sleep and breathing in children. A developmental approach. New York: Marcel Dekker, 2000.

53 O'Brien LM, Holbrook CR, Mervis CB, et al. Sleep and neurobehavioral characteristics of 5- to 7-year-old children with parentally reported symptoms of attention-deficit/hyperactivity disorder. Pediatrics 2003;111:554-63.

54 Ali NJ, Pitson D, Stradling JR. Sleep disordered breathing: effects of adenotonsillectomy on behaviour and psychological functioning. Eur J Pediatr 1996; 155:56-62

55 American Academy of Pediatrics. Clinical practice guideline: diagnosis and management of childhood obstructive sleep apnea syndrome. Pediatrics 2002;109:704-12.

Announcement

The second editon of the RCPH Withholding or Withdrawing Life Sustaining Treatment in Children: A Framewwork of Practice is now available. 